\title{
Sustentabilidade na habitação de interesse social: análise bibliométrica
}

\author{
Sustainability in social housing: bibliometric analysis \\ Sostenibilidad en viviendas de interés social: análisis bibliométrico
}

Recebido: 02/10/2021 | Revisado: 08/10/2021 | Aceito: 09/10/2021 | Publicado: 11/10/2021

\author{
Beatrice Lorenz Fontolan \\ ORCID: https://orcid.org/0000-0002-1245-9009 \\ Universidade Tecnológica do Paraná, Brasil \\ E-mail: fontolanbeatrice@gmail.com \\ Alfredo Iarozinski Neto \\ ORCID: https://orcid.org/0000-0002-3160-5251 \\ Universidade Tecnológica do Paraná, Brasil \\ E-mail: iarozinski@professores.utfpr.edu.br
}

\begin{abstract}
Resumo
A sustentabilidade nas habitações de interesse social vem sendo amplamente discutida devido aos impactos ambientais e sociais, entretanto, este tema apresenta diversas linhas de estudo. Neste sentido, o conhecimento sobre o desenvolvimento sustentável na construção civil pode contribuir para os melhores resultados do setor. Este artigo tem como objetivo destacar as áreas de pesquisa e trabalhos relevantes sobre a sustentabilidade em habitações com o foco na população de baixa renda. Assim, uma análise bibliométrica foi realizada com o suporte das ferramentas VOSviewer e CiteSpace II. Os dados brutos (publicações limitadas ao período de 2017 a 2021) foram extraídos da base Web of Science. Os países europeus apresentaram maior número de publicações, com ênfase na Inglaterra estando na primeira colocação. Foram gerados mapas com os autores e referências que apresentaram maiores interações, destacando os autores Mullins, D., Fitzpatrick, S. e European Commission, e para as obras de Vestergaard e Scanlon, (2014), Mulliner e Smallbone, (2013) e Nicol e Humphreys, (2002). Verificando, portanto, a concordância com as áreas encontradas. Das 12 áreas de pesquisa, "plano de habitação", "avaliação energética" e "conforto adaptativo" foram as áreas mais produtivas, ou seja, com a maioria dos trabalhos publicados. As áreas mais recentes foram "plano de habitação", "circularidade na habitação social" e "modelos preditivos", apresentando-se como tendências de estudo. Um mapa panorâmico das publicações sobre o tema analisado e como se encontram foi exibido, com interligações em redes, instruindo futuros estudos aprofundados em uma área particular de interesse ou como eles se relacionam com uns aos outros.
\end{abstract}

Palavras-chave: Edificação sustentável; Desenvolvimento sustentável; Moradia popular; Construção civil.

\begin{abstract}
The sustainability of social housing has been widely discussed due to environmental and social impacts, however, this topic has several areas of study. In this sense, knowledge about sustainable development in civil construction can contribute to the best results in the sector. This article aims to highlight the areas of research and relevant work on sustainability in housing with a focus on the low-income population. Thus, a bibliometric analysis was performed with the support of VOSviewer and CiteSpace II tools. Raw data (publications limited to the period 2017-2021) were extracted from the Web of Science database. European countries had the highest number of publications, with emphasis on England being in the first place. Maps were generated with the authors and references that showed the greatest interactions, highlighting the authors Mullins, D., Fitzpatrick, S. and European Commission, and for the works of Vestergaard and Scanlon, (2014), Mulliner and Smallbone, (2013) and Nicol and Humphreys, (2002). Therefore, verifying the agreement with the areas found. Of the 12 research areas, "housing plan", "energy assessment" and "adaptive comfort" were the most productive areas, that is, with most published works. The most recent areas were "housing plan", "circularity in social housing" and "predictive models", presenting themselves as study trends. A panoramic map of the publications on the analyzed topic and how they are was displayed, with interconnections in networks, instructing future in-depth studies in a particular area of interest or how they relate to each other.
\end{abstract}

Keywords: Sustainable building; Sustainable development; Popular housing; Construction.

\section{Resumen}

La sostenibilidad en las viviendas de interés social viene siendo ampliamente discutida debido a los impactos ambientales y sociales, sin embargo, este tema presenta varias líneas de estudio. En este sentido, el conocimiento sobre el desarrollo sostenible en la construcción civil puede contribuir a los mejores resultados en el sector. Este artículo tiene como objetivo destacar las áreas de investigación y los trabajos relevantes sobre la sostenibilidad en viviendas con enfoque en la población de bajos ingresos. Así, fue realizado un análisis bibliométrico con el soporte de 
las herramientas VOSviewer y CiteSpace II. Los datos brutos (publicaciones limitadas al período de 2017 al 2021) fueron extraídas de la base Web of Science. Los países europeos presentaron el mayor número de publicaciones, con énfasis en Inglaterra situándose en la primera posición. Fueron generados mapas con los autores y las referencias que presentaron mayores interacciones, destacándose los autores Mullins, D., Fitzpatrick, S. y European Commission, y las obras de Vestergaard; Scanlon, (2014), Mulliner; Smallbone, (2013) y Nicol; Humphreys, (2002). Verificando, por lo tanto, la concordancia con las áreas encontradas. De las 12 áreas de investigación, "plan de vivienda", "evaluación energética" y "confort adaptativo" fueron las áreas más productivas, o sea, con la mayor cantidad de trabajos publicados. Las áreas más recientes fueron "plan de vivienda", "circularidad en viviendas sociales" y "modelos predictivos", presentándose como tendencias de estudio. Fue exhibido un mapa panorámico de las publicaciones sobre el tema analizado y cómo se encuentran, con interconexiones en redes, instruyendo futuros estudios en profundidad en un área particular de interés o cómo se relacionan entre sí.

Palabras clave: Edificación sostenible; Desarrollo sostenible; Vivienda popular; Construcción civil.

\section{Introdução}

Em 1987 o termo “desenvolvimento sustentável” foi publicado no relatório da Comissão Mundial do Meio Ambiente e Desenvolvimento - CMMAD- de Brundtland, conhecido como "Nosso Futuro Comum" (CMMAD, 1988), destacando os impactos da atividade humana. Já na Europa e na América do Norte, os conceitos vêm sendo fundamentados e aprimorados após a segunda guerra mundial (Sugahara et al., 2021).

Desde 1995, o International Council for Research and Innovation in Building and Construction (Conseil International du Bâtiment - CIB) tem estudado prioritariamente sobre o tema, o que resultou em duas publicações mundiais que estabeleceram: "Agenda 21 on Sustainable Construction" (CIB, 1999) e o documento conjunto CIB-UNEP "Agenda on Sustainable Construction in developing countries" (CIB, 2002).

No Brasil, as principais mudanças ocorreram somente a partir da Eco 92 nas diferentes esferas da sociedade, sobretudo no setor da construção civil, por meio do desenvolvimento de estratégias que objetivam minimizar os impactos de suas atividades. Em 2007, criou-se o Conselho Brasileiro de Construção Sustentável (CBCS, 2007) com o objetivo de contribuir para a geração e difusão de conhecimento e de boas práticas de sustentabilidade na construção civil. Desde então, o interesse da Academia sobre a temática tem aumentado progressivamente, face a essa realidade que tem assolado todo o planeta.

Além disso, o setor da construção civil tem se reconfigurado na busca de reavaliar e mitigar o uso dos recursos naturais, as emissões de gases e resíduos. Deste modo, a sustentabilidade e o desenvolvimento sustentável vem sendo amplamente discutidos, haja visto a elevada preocupação com a extensão dos danos causados pelo homem, com a sua reparação, bem como com projetos de menor impacto ambiental, sem comprometer a economia e buscando uma sociedade justa.

A sustentabilidade apresenta uma visão ampla e sistêmica, tradicionalmente configurada em três pilares principais: social, econômico e ambiental, com o objetivo de encontrar o equilíbrio entre eles (Sattler, 2007). Entretanto, segundo Ignacy Sachs (Sachs, 2002), existem oito dimensões da sustentabilidade que devem ser consideradas, podendo ser aprofundado, para abranger vários aspectos do meio ambiente e de seu entorno, partindo da premissa de que o desenvolvimento deve transcender o significado econômico, visto ser a área mais discutida. O autor classifica em ecológica, econômica, social, cultural, ambiental, territorial, política nacional e internacional.

No que se refere à construção do empreendimento, é sustentável quando as atividades são direcionadas de forma a permitir a redução dos impactos ambientais e à saúde decorrentes da obra, das edificações e do ambiente externo construído (Ihuah et al., 2014).

A habitação de interesse social (HIS) visa o atendimento às necessidades de habitação à população de baixa renda, sendo suas particularidades relacionadas ao momento histórico e às características socioeconômicas, políticas e culturais do local. No entanto, há evidências que mostram que a habitação social não é adequadamente apoiada para alcançar objetivos 
sustentáveis, apesar de sua importância para enfrentar a crise habitacional (Oyebanji et al., 2017).

Lutzkendorf e Lorenz (2005) afirmam que para classificar conjuntos habitacionais de interesse social sustentável, é necessário a proteção do ambiente natural, dos recursos naturais básicos, da saúde e bem-estar humanos, dos valores sociais e dos bens públicos, e proteção e preservação de bens de capital e materiais. Deste modo, a HIS sustentável não pode ser pensada exclusivamente como a possibilidade de uso adequado dos recursos naturais, mas deve incluir todas as questões relacionadas aos três pilares de forma sistêmica, proporcionando a satisfação dos residentes e considerando os vários aspectos pertinentes à produção de projetos habitacionais (Reis \& Lay, 2010).

É valido destacar que, o desenvolvimento sustentável é uma prática que integra vários critérios, incluindo, por exemplo, eficiência energética, durabilidade, minimização de resíduos, impacto social, bons ambientes internos, durabilidade, controle de poluição, avaliação do ciclo de vida, convivialidade e conforto do usuário. Assim, o impacto da construção e operação de edifícios em questões de sustentabilidade é significativo (Olanrewaju et al., 2018). Portanto, o tema pode ser amplamente discutido com o foco em áreas distintas.

Nesse contexto, o presente artigo tem como objetivo apontar as tendencias das áreas de pesquisa, bem como os autores e trabalhos relevantes sobre a sustentabilidade na HIS. Busca-se responder aos seguintes questionamentos: Quais as principais áreas de pesquisa? Quais as tendências de estudos atuais? Onde estão as áreas mais ativas? Quais os artigos chave?

\section{Metodologia}

A abordagem metodológica deste estudo consiste em uma revisão sistemática qualitativa com abordagem quantitativa da literatura (Gil, 2002), as análises são apropriadas para mapear, avaliar e sintetizar literatura para desenvolver o conhecimento em um campo (Tranfield et al., 2003). Além disso, o método permite a identificação de lacunas na pesquisa e serve para o desenvolvimento de novas agendas de pesquisa.

A bibliometria consiste na aplicação de técnicas estatísticas e matemáticas para descrever aspectos da literatura, utilizando métodos que quantificação da literatura acadêmica disponível, ao contrário da pesquisa bibliográfica tradicional, busca uma avaliação objetiva e qualitativa (Spinak, 1996). Estes métodos vêm sendo utilizados para rastrear as citações de revistas acadêmicas e ajudam na organização e análise de grandes quantidades de informações, auxiliando por meio de análises estatística e matemática para identificar padrões (Daim et al., 2006).

Neste estudo bibliométrico utilizou-se da análise descritiva, a análise de cocitações, coocorrência de termos e análise cluster. Foram selecionadas referências sobre a aplicação da sustentabilidade nas áreas relacionadas a construção civil, com foco nas HIS. Utilizou-se da seguinte string de busca: "social housing" or "affordable housing" and "sustainab*" A busca foi realizada na base Web of Science (WoS) - Coleção Principal (Clarivate Analytics), selecionando apenas articles e reviews publicados no período de 2017 a 2021. Retornaram desta busca 933 publicações.

Foram importados os metadados (título, resumo, autores, palavras-chave, periódico, referências e número de citações) destes artigos. A escolha da base consiste no fato de ser a principal fonte de dados para o CiteSpace II e, de acordo com Chadegani et al. (2013), a WoS contempla periódicos com maior fator de impacto quando comparado àqueles abrangidos pela Scopus (Elsevier). Além disso, é realizada a análise das referências dos artigos, que possibilita ampliar o acesso à livros, relatórios e periódicos de outras bases.

Os dados importados da WoS foram processados no software bibliométrico CiteSpace II e no VOSviewer 1.6.16, projetados para facilitar a visualização das respostas sobre estrutura e dinâmica de um conjunto de registros bibliográficos de publicações relevantes. O software desenhou as redes que se formaram, utilizando técnicas de mapeamento de agrupamento de dados. Cada ponto representa um nó (citação), os quais estão conectados por links de cocitações (linhas que conectam os nós). Assim, as referências, autores e termos que apareceram com rótulos se referem a trabalhos de destaque. 


\section{Resultados e Discussão}

\subsection{Análise descritiva}

Tratando-se da quantidade de periódicos publicados por países (Figura 1), a plataforma WoS detectou os dez países mais relevantes na pesquisa sobre o tema, mostrando as regiões onde o tema habitação sustentável tem tido mais publicações nas bases de dados levantadas. Destaca-se a Inglaterra em primeiro lugar com 139 publicações seguido da Espanha com 103, e o Brasil em sexto lugar com 50 publicações.

Figura 1. Publicações por países.

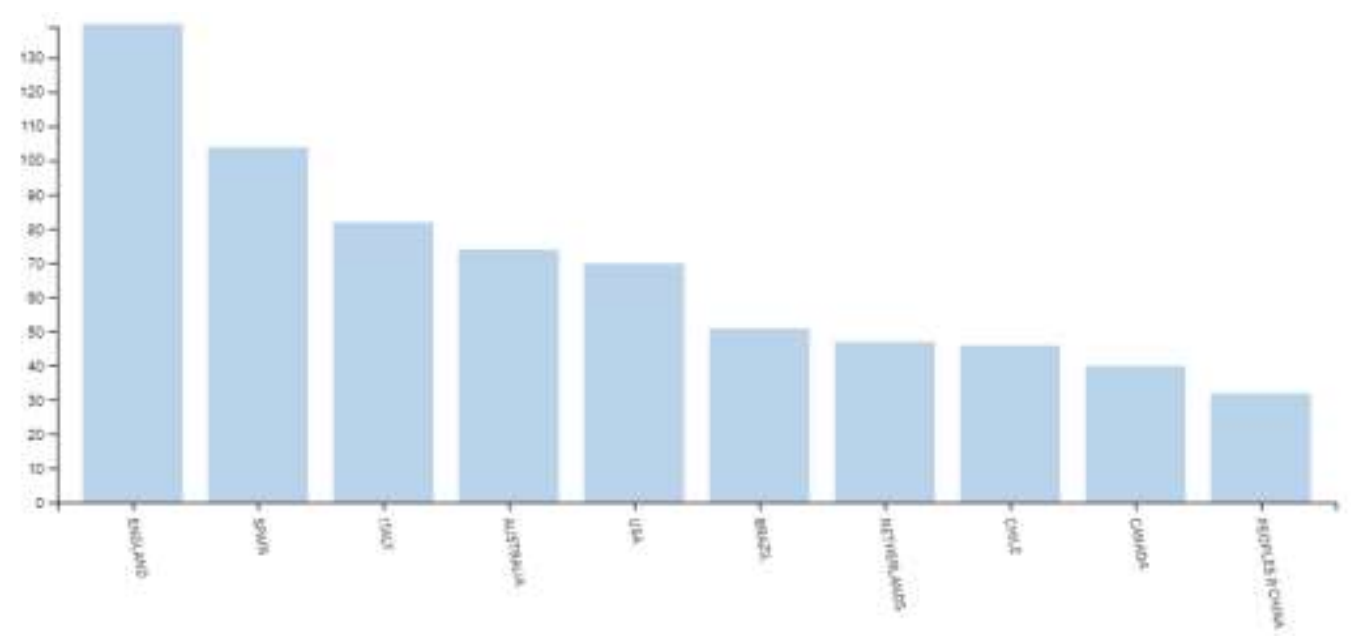

Fonte: Scopus (2021).

No que diz respeito à quantidade de trabalhos publicados entre os anos de 2017 e 2021, a Figura 2 apresenta a evolução das publicações ao longo dos anos, em que é perceptível o crescimento dos temas na literatura. Em 2020 foi observado o maior número, totalizando 241 publicações e há uma tendência de crescimento visto que até o mês de junho de 2021 constam 71 artigos.

Figura 2. Distribuição anual dos artigos.

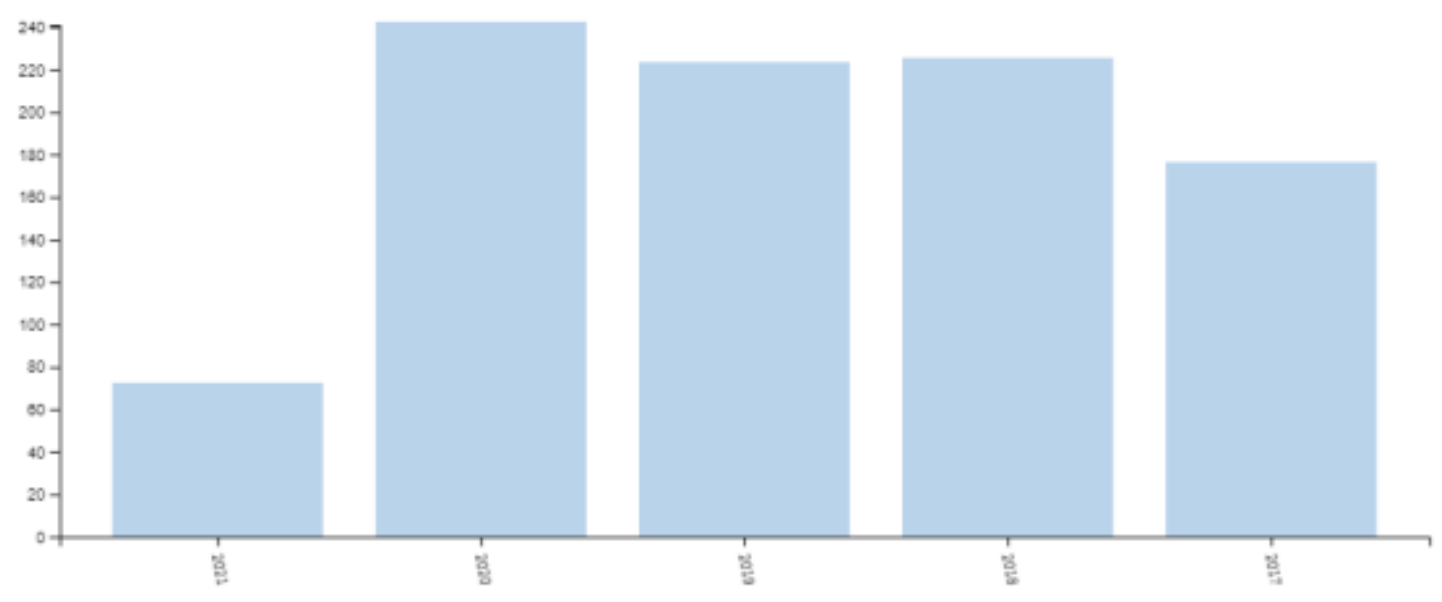

Fonte: Scopus (2021)

\subsection{Análise das co-citações de autores}

Balancieri et al. (2005) afirmam que, as pesquisas científicas atingem amplitude acadêmica e social, quando os pesquisadores se relacionam entre si, o que gera uma rede de publicações e, consequentemente, promove o aumento da 
qualidade dos resultados das pesquisas.

Nesse sentido, quanto mais vezes dois trabalhos são concomitantemente citados, maior sua probabilidade de apresentarem conteúdos associados (Spinak, 1996). Assim, há similitude de conteúdo entre os citados quando referenciados juntos em uma terceira pesquisa e torna diretamente proporcional a frequência de cocitação com a relação de proximidade entre eles.

Nesta pesquisa quando relacionam os autores com no mínimo 20 citações, o VOSviewer selecionou 80 autores que são citados mais frequentes nestes documentos. Uma característica relevante do mapa gerado é o diâmetro dos círculos, que aumenta de acordo com a frequência de ocorrência das citações. Considera-se que a busca ocorre ao se estimar os autores mais recorrentes relacionados a um campo do conhecimento (princípio da Lei de Zipf).

Posteriormente, os documentos foram relacionados às suas referências, construindo-se uma rede de acoplamentos bibliográficos. Para formar esse mapa com referências acopladas, o VOSviewer selecionou autores que foram referenciados ao menos três vezes por mais de um autor. Os autores com maiores interações são, Mullins, Fitzpatrick e European, Commission. Assim, por meio da Figura 3 verificou-se à presença de clusteres (diferentes cores da figura), é possível identificar a relação entre autores, que na maioria dos casos fundamentam a construção do pensamento científico estabelecendo relações entre si, formando uma rede coesa e conectada.

Figura 3. Rede de cocitações de

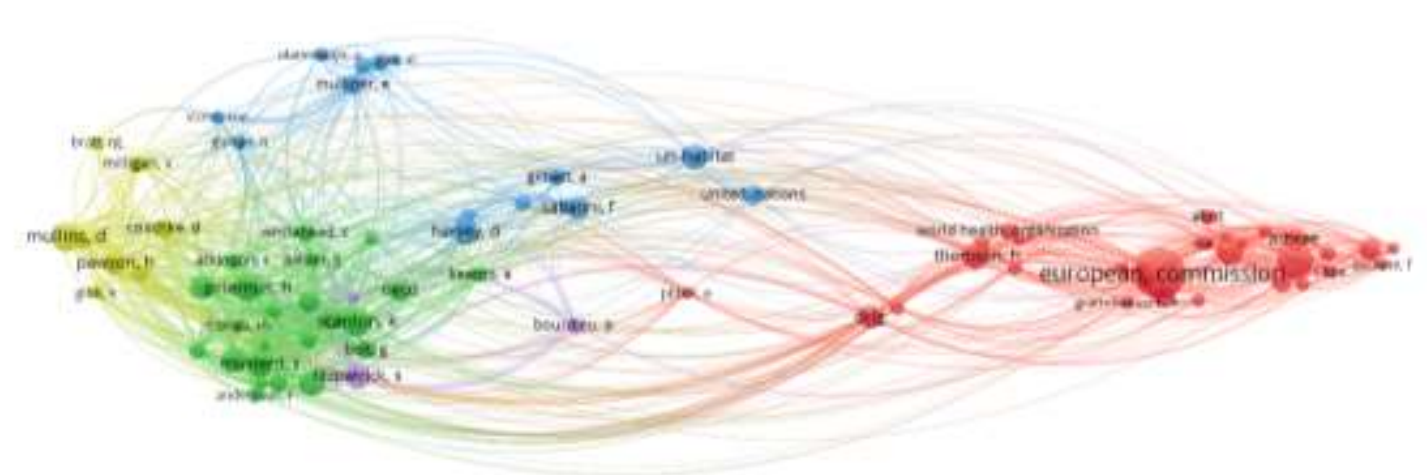

autores.

Fonte: VOSviewer (2021).

\subsection{Análise das cocitações de documentos}

A Figura 4 apresenta os documentos mais citados conjuntamente pelos artigos da área de pesquisa. Para a análise da amostra estudada, foi determinado o valor mínimo de 7 citações por referência, nos quais foram identificadas 118 bibliografias que atendiam aos requisitos da pesquisa. Destaque para as obras de Vestergaard e Scanlon, (2014) sobre habitações de cunho social na Europa, Mulliner e Smallbone, (2013) sobre método de multicritério para habitações sustentáveis de baixo custo e Nicol e Humphreys, (2002) sobre conforto térmico e sustentabilidade em edifícios. 
Figura 4. Rede de cocitações de documentos.

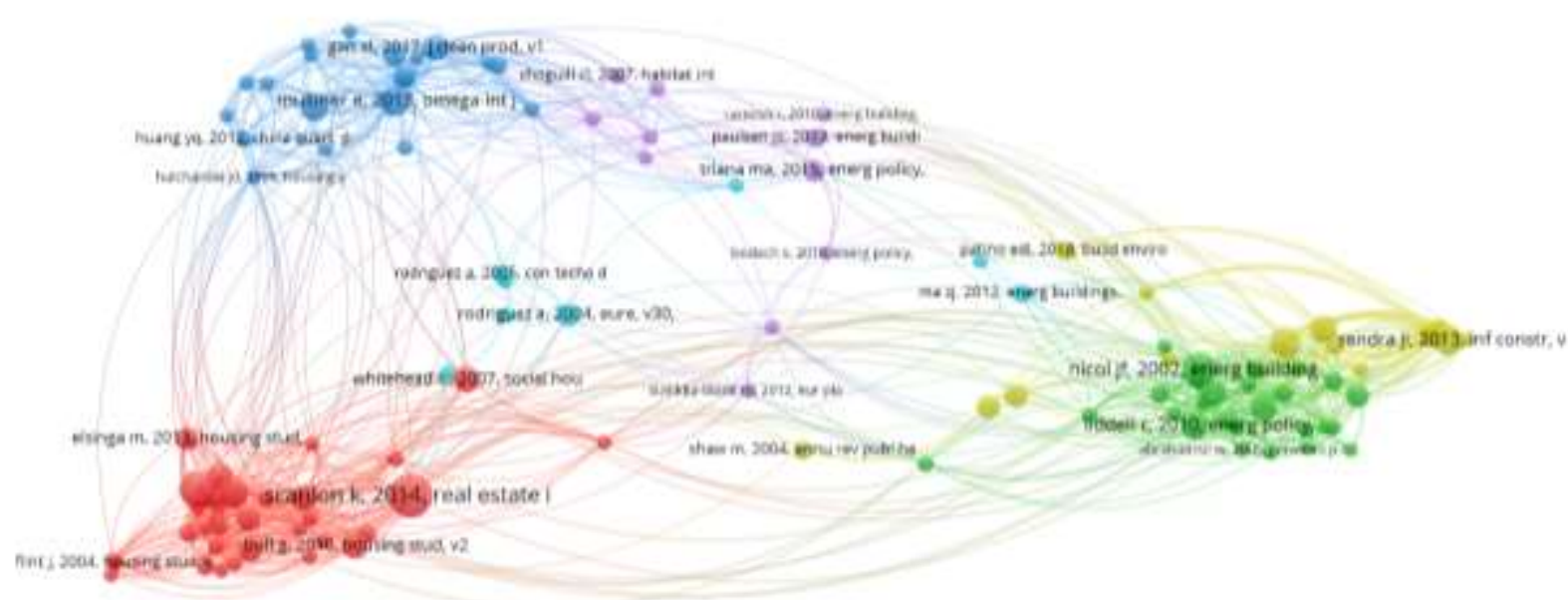

A. vosuiewer

Fonte: VOSviewer (2021).

\subsection{Principais áreas de pesquisa}

A coocorrência de termos do título e do resumo implica na importância e a proximidade de conceitos e métodos utilizados pelos artigos (itens). Ademais, é possível verificar a escala de tempo da análise por meio de um gradiente de cores, observando os termos mais recentes

A Figura 5 mostra as palavras-chaves de maior ocorrência nos títulos e resumos ao avaliar as publicações encontradas no período analisado. Utilizando a indicação de no mínimo 40 ocorrências de uma palavra-chave, foram encontrados 101 termos, destes, foram selecionados os 61 mais relevantes para a formação do mapa. Dessa forma, os principais nós são development, process, city, building e use. A rede de co-ocorrência de termos mostra uma maior preocupação das pesquisas com a política habitacional (government, housing policy, policy, city, developement) e com a eficiência energética das habitações (confort, energy efficiency, energy consumation, performance). Como métodos preponderantes de pesquisa se destacam o Survey e o estudo de caso. 
Figura 5. Rede de co-ocorrência de termos.

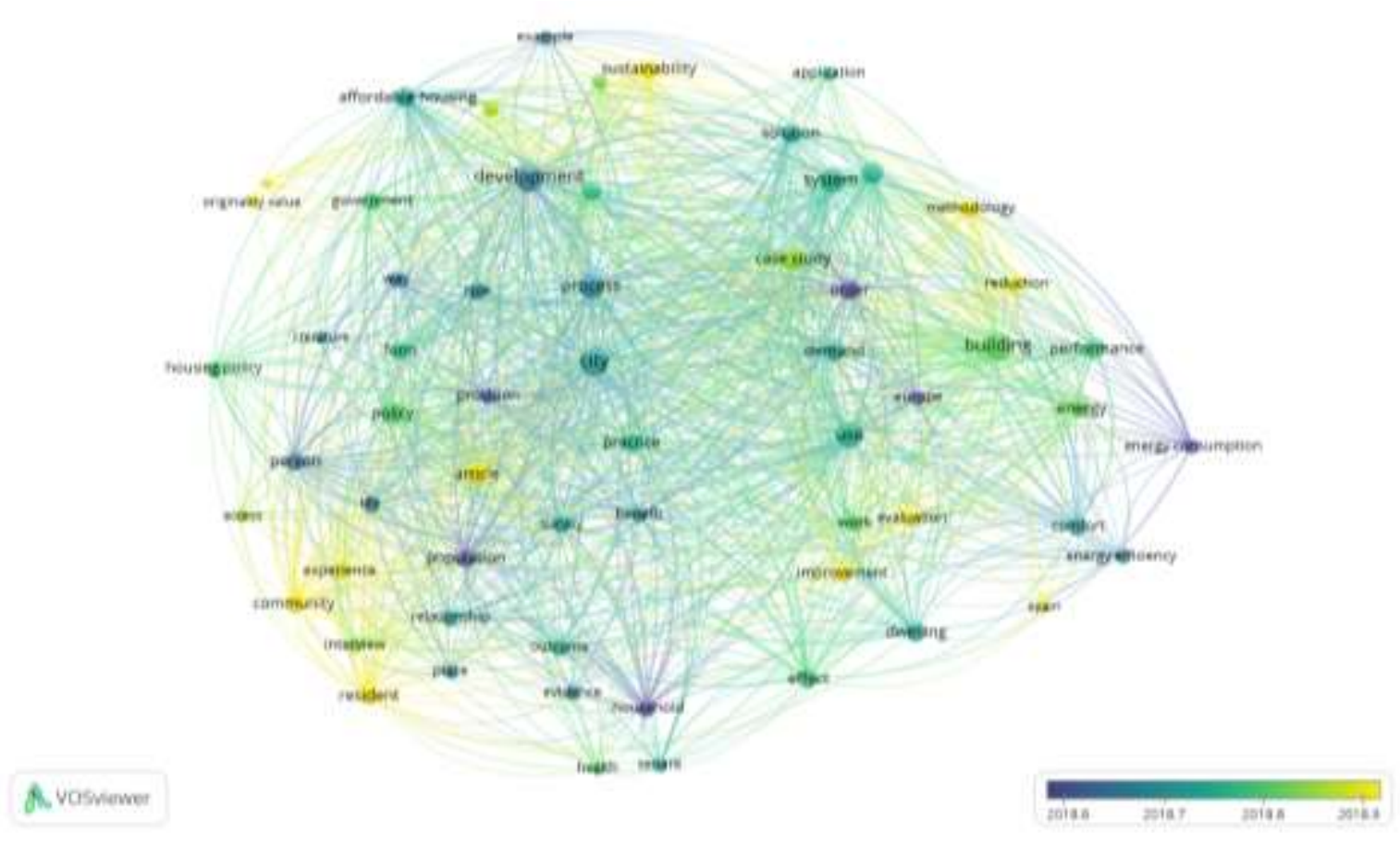

Fonte: VOSviewer (2021).

Com o auxílio do CiteSpace II, foram agrupadas as referências dos artigos (dados de entrada) em 12 clusters principais, os quais representam as áreas de pesquisa de maior destaque. Nota-se na Figura 6 que a maior área (cluster \#0, em vermelho, com o maior número de obras) é a housing plan, seguida do household energy poverty perspective (cluster \#1), adaptive comfort (cluster \#2) e circular social housing (cluster \#3), e assim sucessivamente até indoor environmental quality (cluster \#11). Os clusters rotulados apontam o contexto em que são mais citados, sendo os termos dos rótulos extraídos de títulos dos artigos que citaram o trabalho.

Figura 6. Principais áreas de pesquisa.

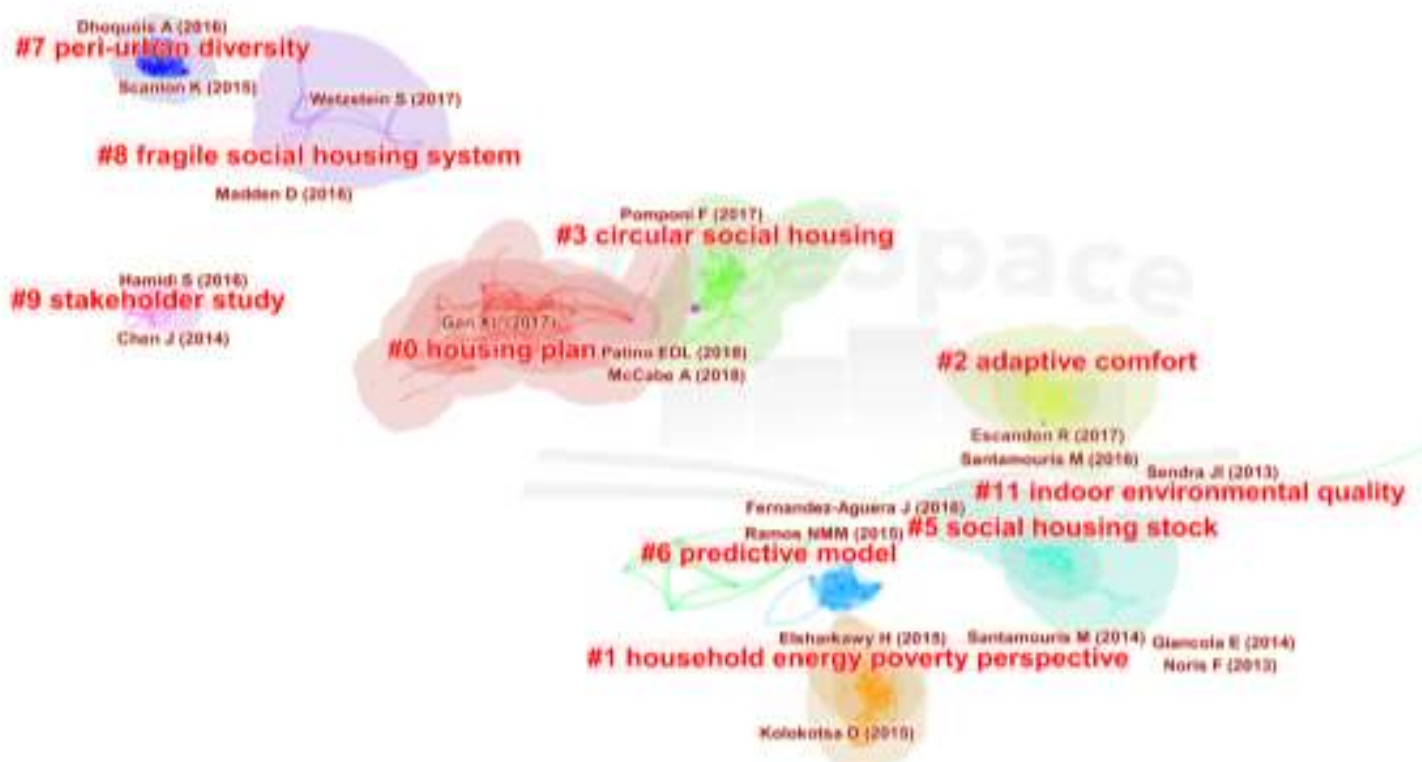

Fonte: CiteSpace (2021). 
A modularidade Q e a silhueta significativa de pontuações são duas métricas importantes que fornece os resultados sobre as propriedades estruturais globais da rede. A modularidade de uma rede mede a extensão em que uma rede pode ser decomposta em vários componentes, ou módulos. Nesta base de dados, a modularidade Q é 0,7896 relativamente elevada, o que significa que a rede está razoavelmente dividida em clusters de baixo acoplamento. O valor de uma silhueta de um cluster mede a qualidade de configuração de cluster. A silhueta significativa de pontuação de 0,9157 sugere que a homogeneidade destes aglomerados é elevada (Chen, 2014). A Tabela 1 mostra as referências mais citadas em cada cluster.

Tabela 1. Obras mais citadas dos clusters gerados da pesquisa.

\begin{tabular}{|c|c|c|c|}
\hline Autores & Ano & Título & Cluster \\
\hline Gan, XL. et al. & 2017 & $\begin{array}{l}\text { How affordable housing becomes more sustainable? A stakeholder } \\
\text { study }\end{array}$ & Housing plan \\
\hline $\begin{array}{l}\text { Kolokotsa, D. e } \\
\text { Santamouris, M. }\end{array}$ & 2015 & $\begin{array}{l}\text { Review of the indoor environmental quality and energy consumption } \\
\text { studies for lowincome households in Europe }\end{array}$ & $\begin{array}{l}\text { Household energy } \\
\text { poverty perspective }\end{array}$ \\
\hline Santamouris M. & 2016 & $\begin{array}{l}\text { Innovating to zero the building sector in Europe: Minimising the } \\
\text { energy consumption, eradication of the energy poverty and mitigating } \\
\text { the local climate change }\end{array}$ & Adaptive comfort \\
\hline $\begin{array}{l}\text { Pomponi F. e } \\
\text { Moncaster, A. }\end{array}$ & 2017 & Circular economy for the built environment: A research framework & $\begin{array}{l}\text { Circular social } \\
\text { housing }\end{array}$ \\
\hline Sendra, J. J. et al. & 2013 & $\begin{array}{l}\text { Energy intervention in the residential sector in the south of Spain: } \\
\text { Current challenges }\end{array}$ & Social housing stock \\
\hline $\begin{array}{l}\text { Fernández- } \\
\text { Agüera, J. et al. }\end{array}$ & 2016 & $\begin{array}{l}\text { An approach to modelling envelope airtightness in multi-family social } \\
\text { housing in Mediterranean Europe based on the situation in Spain }\end{array}$ & Predictive model \\
\hline Scanlon, K. et al. & 2015 & Social housing in Europe & Peri-urban diversity \\
\hline Wetzstein, S. & 2017 & The global urban housing affordability crisis & $\begin{array}{l}\text { Fragile social } \\
\text { housing system }\end{array}$ \\
\hline $\begin{array}{l}\text { Chen, J. e Deng, } \\
\text { L. }\end{array}$ & 2014 & $\begin{array}{l}\text { Financing Affordable Housing Through Compulsory Saving: The Two- } \\
\text { Decade Experience of Housing Provident Fund in China }\end{array}$ & Skateholder study \\
\hline Giancola, E. et al. & 2014 & $\begin{array}{l}\text { Evaluating rehabilitation of the social housing envelope: Experimental } \\
\text { assessment of thermal indoor improvements during actual operating } \\
\text { conditions in dry hot climate, a case study }\end{array}$ & $\begin{array}{l}\text { Indoor environmental } \\
\text { quality }\end{array}$ \\
\hline
\end{tabular}

Fonte: Autores (2021).

\subsection{Origem e desenvolvimento histórico das áreas de pesquisa}

Foi feita a análise visual da linha do tempo da rede com o auxílio do CiteSpace II, para compreender a origem, o desenvolvimento histórico e o estado atual dos clusters (áreas de pesquisa). A divisão é organizada por cluster em uma linha horizontal crescente da esquerda para a direita. Figura 7 observa-se que as áreas de pesquisa com estudos mais recentes (próximos de 2020$)$ e promissores são: housing plan $(\approx 2018)$, circular social housing $(\approx 2017)$ e predictive model $(\approx 2016)$. Áreas como fragile social housing system e indoor enviroment quality, por exemplo, há muito (desde $\approx 2015$ e $\approx 2013$, respectivamente) não apresentam volume de publicações, o que evidencia uma estagnação do tema. 


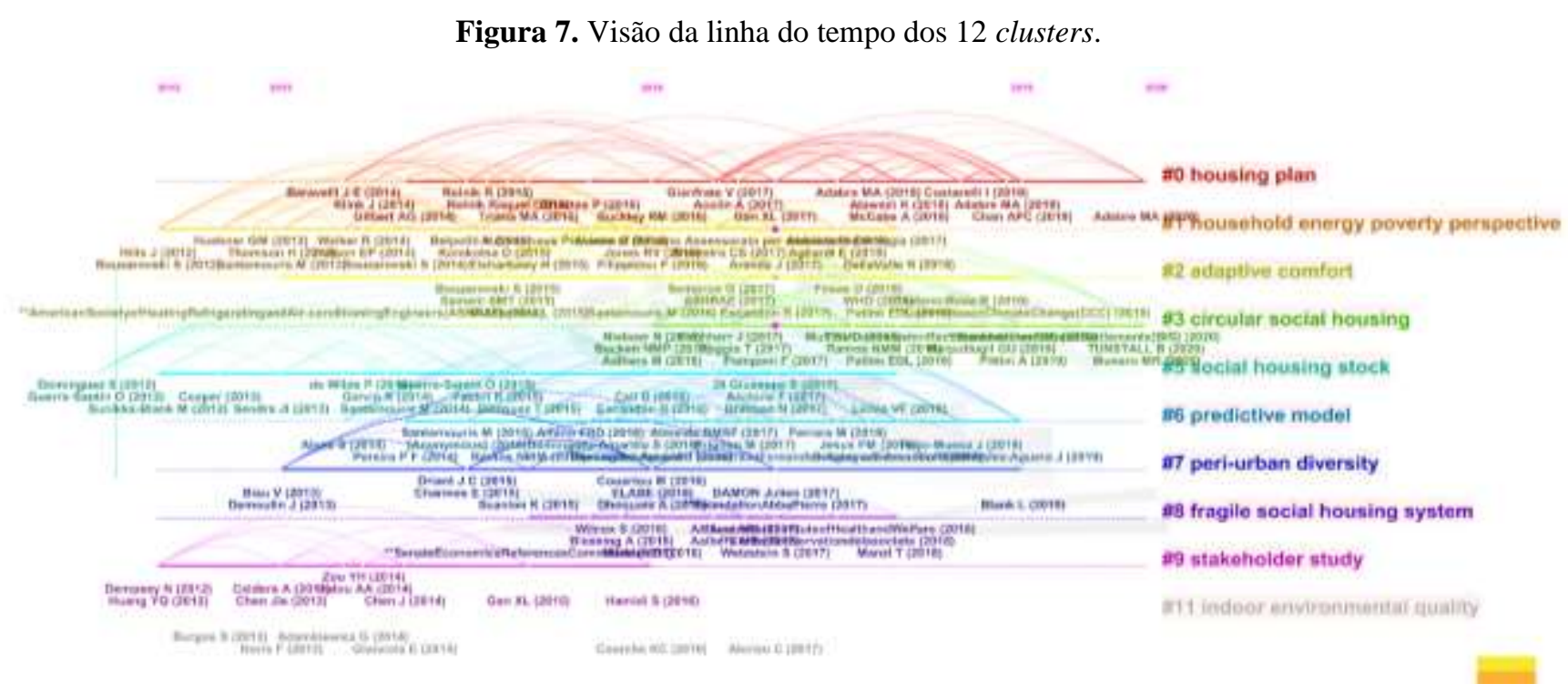

Fonte: CiteSpace (2021).

\section{Conclusão}

Os estudos sobre sustentabilidade na HIS são mais recorrentes nos países europeus, e vem crescendo ao longo dos últimos cinco anos, mostrando ser um tema promissor. Com o auxílio dos softwares VoSviewer foi possível observar a rede de cocitações de autores, com destaque para Mullins, D., pesquisador da área de empreendimento social e comportamento organizacional, Fitzpatrick, S., que aborda sobre exclusão de habitação, políticas de bem-estar, oferta de habitação e as políticas públicas da Comissão Europeia, que também se mostraram como base das pesquisas.

Ademais, dentre os documentos mais citados conjuntamente pelos artigos, estão as obras de Vestergaard e Scanlon, (2014), Mulliner e Smallbone, (2013) e Nicol e Humphreys, (2002), que estudam as habitações sociais na Europa, critérios de decisão para HIS sustentáveis e análise do conforto e eficiência energética nas habitações, respectivamente. Analisando-se a linha do tempo e a rede de palavras, essa tendência é confirmada, na medida em que as três áreas mais recentes e promissoras foram o planejamento urbano, modelo preditivo e circularidade na HIS.

Por meio do CiteSpace II foram identificadas 12 áreas de pesquisa e o panorama atual que evidencia os temas mais promissores e o surgimento (interações significativas) de novas áreas emergentes nos últimos anos. É perceptível a preferência por temas voltados ao planejamento urbano, eficiência energética e conforto.

Dessa forma, foi obtido um mapa panorâmico das principais publicações sobre o tema analisado e autores e de como elas estão interligadas em redes, integrando e formando áreas de pesquisa. O presente artigo, portanto, auxilia e serve, também, como estudo exploratório para trabalhos futuros de investigação em profundidade sobre determinada área de interesse ou, ainda, sobre como áreas ou publicações se ligam e/ou se relacionam.

Sugere-se para trabalhos futuros uma pesquisa mais ampla em várias plataformas de base de dados e utilização e a análise bibliométrica de cada área apresentada para saber o estado da arte delas e aprofundar os conhecimentos.

\section{Referências}

Balancieri, R., Bovo, A. B., Kern, V. M., Pacheco, R. C. S. \& Barcia, R. M. (2005) A análise de redes de colaboração científica sob as novas tecnologias de informação e comunicação: um estudo na Plataforma Lattes. Ciência da Informação, 34(1), 64-77.

Chadegani, A. C, Salehi, H., Yunus, M., Ferhadi, H., Fooladi, M., Farhadi, M. \& Ebrahim, N. A. (2013). A comparison between two main academic literature collections: Web of Science and Scopus databases. Asian Social Science, Brasília, 9(5), 18-26.

Chen, C. (2014). The CiteSpace manual. College of Computing and Informatics. http://cluster.cis.drexel.edu/ cchen/citespace/ 
Research, Society and Development, v. 10, n. 13, e267101321338, 2021

(CC BY 4.0) | ISSN 2525-3409 | DOI: http://dx.doi.org/10.33448/rsd-v10i13.21338

CIB. (2002). Agenda 21 for Sustainable Construction in Developing Countries - a discussion document. Conseil International du Bâtiment. Pretoria: IB \& UNEP-ITEC.

CIB. (1999). Agenda 21 on Sustainable Construction. Conseil International du Bâtiment. Netherlands: CIB Report Publication 237.

CBCS. (2007). Missão, Visão, Origem. Conselho Brasileiro de Construção Sustentável (CBCS). http://www.cbcs.org.br/website/institucional/sho w.asp?ppgCode=BCCF20BC-8628-4D3D-83ED-FBA37CFA560D.

Daim, U. T., Rueda U., Martin, H. \& Gerdsri, P. (2016). Forecasting emerging technologies: use of bibliometrics and patent analysis. Technological Forecasting and Social Change Journal, Nova York, 73(8), 981-1012.

Gil, Antonio Carlos. (2002). Como elaborar projetos de pesquisa. Atlas

Ihuah, P. W., Kakulu, I. I. \& Eaton, D. (2014). A review of critical project management success factors (CPMSF) for sustainable social housing in Nigeria. International Journal of Sustainable Built Environment, 3(1), 62-71.

Lutzkendorf, T. \& Lorenz, D. (2005). Sustainable property investment: valuing sustainable buildings through property performance assessment. Building Research \& Information, 33(3), 212-234.

Mulliner, E., Smallbone, K. \& Maliene, V. (2013). An assessment of sustainable housing affordability using a multiple criteria decision making method. Omega, 41(2), 270-279.

Nicol, J. F. \& Humphreys, M. A. (2001). Adaptive thermal comfort and sustainable thermal standards for buildings. Energy and Buildings, 34(6), 563-572.

CMMAD (1998). Comissão Mundial Sobre Meio Ambiente e Desenvolvimento. Nosso Futuro Comum. FGV.

Olanrewaju, A. L., Tan, S. Y. \& Abdul-Aziz, A. R. (2018). Housing providers' insights on the benefits of sustainable affordable housing. Sustainable Development, 26(6), 847-858.

Oyebanji, A. O., Liyanage, C. \& Akintoye, A. (2017). Critical success factors (CSFs) for achieving sustainable social housing (SSH). International Journal of Sustainable Built Environment, 6(1), 216-227.

Reis, A. T. L. \& Lay, M. C. D. (2010). O projeto da habitação de interesse social e a sustentabilidade social. Ambiente Construído, Porto Alegre, 10(3), 99119.

Sachs, I. (2002). Caminhos para o desenvolvimento sustentável. (2a ed.), Editora Garamond.

Sattler, M. A. (2007). Habitações de baixo custo mais sustentáveis: a casa Alvorada e o Centro Experimental de tecnologias habitacionais sustentáveis. Porto Alegre: ANTAC.

Spinak, E. (1996). Dicionário enciclopédico de bibliometria, cienciometria e informetria. Unesco.

Sugahara, E. S., Freitas, M. R. De \& Cruz, V. A. L. da. (2021). Análise das certificações ambientais de edificações: AQUA, PROCEL, LEED e Casa Azul. Interação - Revista de Ensino, Pesquisa e Extensão, Varginha, 23(1), 12-24. https://periodicos.unis.edu.br/index.php/interacao/article/view/285.

Tranfield, D., Denyer, D. \& Smart, P. (2003). Towards a methodology for developing evidence-informed management knowledge by means of systematic review. British Journal of Management, 14(3), 207-222. https://doi.org/10.1111/1467-8551.00375.

Vestergaard, H. \& Scanlon, K. (2014). Social housing in Denmark. Social housing in Europe: John Wiley \& Son, 77-89. 